\title{
Carcinoma of the prostate: remission of paraparesis with inhibitors of bone resorption
}

\author{
R.C. Percival, M.E. Watson, J.L. Williams and J.A. Kanis \\ University Department of Human Metabolism \& Clinical Biochemistry, Royal Hallamshire Hospital, Sheffield \\ $S 102 R X, U K$.
}

\begin{abstract}
Summary: We describe a patient with metastatic carcinoma of the prostate associated with paraplegia. The patient also had Paget's disease of bone elsewhere. Because the neurological lesion was thought to be due to Paget's disease, the patient was treated with inhibitors of bone resorption. Treatment rapidly induced clinical remission and inhibition of bone resorption, and withdrawal was associated with relapse. This suggests that such agents may be of value in the treatment of bone disease of prostatic carcinoma.
\end{abstract}

\section{Introduction}

Carcinoma of the prostate commonly gives rise to osteoblastic bony metastases. Bone formation is increased, as judged by bone histology or indirectly by serum activity of akaline phosphatase. Less well appreciated is that bone resorption is also increased (Mundy, 1979) so that skeletal turnover is augmented. We report here a patient with prostatic bone disease and spinal paraparesis due to vertebral collapse, and his remission following medical treatment with inhibitors of bone turnover.

\section{Case report}

A 78 year old male was admitted to hospital with a history of mid-lumbar back pain and progressive weakness of both legs over the previous 3 weeks. He gave no recent history of bowel or bladder disturbance, but $2 \mathrm{y}$ previously he had a retropubic prostatectomy for benign prostatic hypertrophy confirmed on subsequent histological examination. On examination, he had a flaccid paraparesis (grade 2-3) with sensory impairment below T11 involving the 'saddle' area. He was tender over the lower thoracic spine. On rectal examination, a small prostatic remnant was palpable.

Radiographs of the pelvis and thoracolumbar spine showed osteosclerosis and enlargement of the left ischiopubic ramus and the vertebral bodies of T9 and L1. Vertebral collapse of T11 was noted and a myodil myelogram showed a complete block at its lower

Correspondence: J. A. Kanis, F.R.C.P., M.R.C.Path. Accepted: 12 September 1984 border (Figure 1). A clinical diagnosis of paraparesis secondary to Paget's disease was made, which was supported by the biochemical finding of increased bone turnover as judged by urinary excretion of hydroxyproline and increased serum alkaline phosphatase (Figure 2).

The patient was treated with oral clodronate (dichloromethylene diphosphonate) for 3 months and an 8 day course of mithramycin $(800 \mu \mathrm{g}$ given daily i.v. in $500 \mathrm{ml} \mathrm{5 \%}$ dextrose). The paraparesis improved and values for timed-straight leg raising increased from 0 to $10 \mathrm{~s}$ by 1 week and to greater than $50 \mathrm{~s}$ by the first month. At that time he was able to walk with the aid of a Zimmer frame. Biochemical assessment showed a marked fall in bone resorption as judged by urinary hydroxyproline/creatinine and fasting calcium excretion, together with a reduction in plasma calcium (Figure 2). Two months after the start of treatment the patient was walking with a stick and a repeat myelogram showed free flow of myodil past the previous site of obstruction (Figure 1).

Three months after stopping treatment, the patient deteriorated with recurrence of lower thoracic back pain and progressive paraplegia. A myelogram at this stage showed a complete block at T11. Biochemical investigations showed that bone turnover had increased once more (Figure 2).

A decompressive laminectomy was performed. At operation, an adherent mass was found over the dura at T10-11 on the right, compressing the spinal cord. Histological examination showed evidence of a moderately differentiated adenocarcinoma of prostatic origin infiltrating adjacent osteosclerotic bone.

Following surgery, the patient made a full and rapid

(C) The Fellowship of Postgraduate Medicine, 1985 


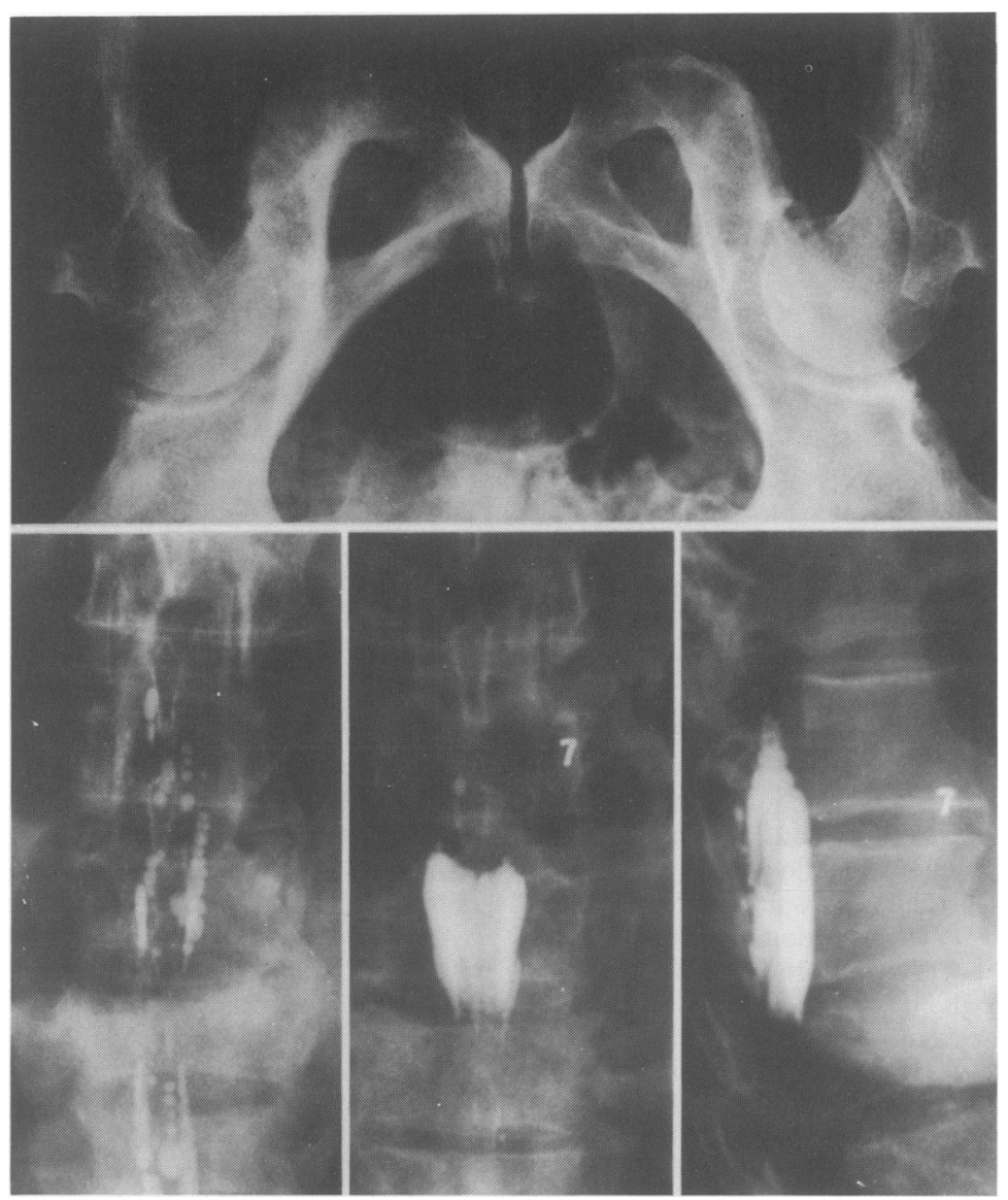

recovery with complete resolution of the paraparesis. Two months later he underwent orchidectomy (Figure 2 ) and has maintained his remission now for $2 y$.

\section{Discussion}

Clodronate and mithramycin are potent inhibitors of bone turnover used in the treatment of Paget's disease (Kanis \& Russell, 1982) and both have been used successfully in the treatment of progressive spinal cord compression due to Paget's disease (Douglas et al., 1981).
This patient, initially thought to have spinal cord dysfunction due to Paget's disease, showed a marked and rapid biochemical, radiographic and clinical response similar to that observed in Paget's disease. Following relapse of the patient's paraparesis after stopping treatment, he was found to have metastatic carcinoma of the prostate.

This is the first demonstration of improvement in spinal cord dysfunction due to metastatic carcinoma of the prostate following treatment with inhibitors of bone turnover. The effect was biphasic, suggesting activity of both agents, mithramycin having the more rapid onset of effect. The mechanism of action of these 


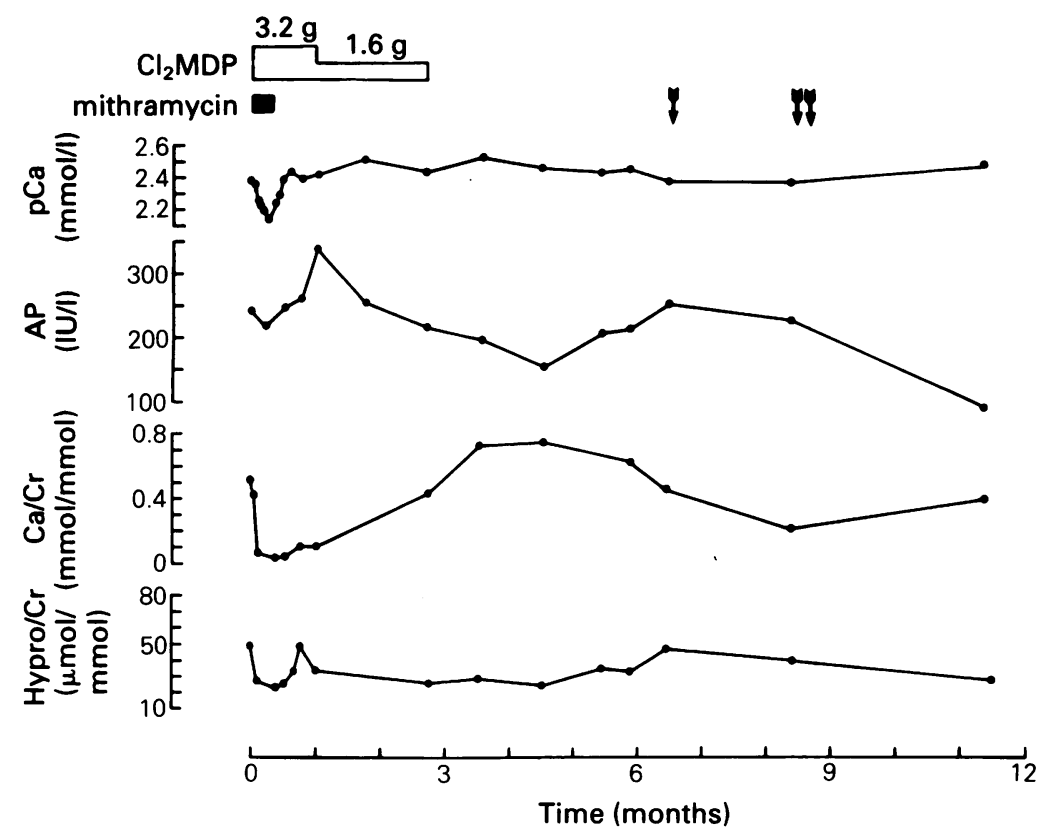

Figure 2 Biochemical responses to treatment with mithramycin and dichloromethylene diphosphonate $\left(\mathrm{Cl}_{2} \mathrm{MDP}\right)$. There was a biphasic fall in bone resorption as judged by urinary hydroxyproline/creatinine ratio (Hypro/Cr: NR $<30 \mathrm{mmol} / \mathrm{mmol}$ ) and bone formation judged by serum alkaline phosphatase (AP: NR $<100 \mathrm{IU} /$ 1.). Net bone resorption (fasting urinary calcium/creatinine ratio; $\mathrm{Ca} / \mathrm{Cr}: \mathrm{NR}<0.4 \mathrm{~mol} / \mathrm{mol}$ ) and plasma calcium (pCa) also decreased. Note the rise in alkaline phosphatase, hydroxyproline and calcium/creatinine ratio indicating relapse before decompressive laminectomy $(\downarrow)$ and the fall in these indices of bone turnover following treatment with cyproterone and orchidectomy $(\downarrow \downarrow)$.

agents is uncertain. Mithramycin is a cytoxic agent, but the doses used in our patient, recommended for Paget's disease are considerably lower than those used for tumour chemotherapy (Heath, 1981). The rapid response to treatment suggests that treatment decreased bone blood flow through affected tissue, thereby improving blood supply to the ischaemic cord, a mechanism contributing to the improvements of cord compression syndromes in Paget's disease (Douglas et al., 1981; Herzberg \& Bayliss, 1980). Irrespective of the mechanism of action, treatment was associated not only with marked clinical improvement, but with biochemical evidence of

\section{References}

DOUGLAS, D.L., DUCKWORTH, T., KANIS, J.A., JEFFERSON, A.A., MARTIN, T.J. \& RUSSELL, R.G.G. (1981). Spinal cord dysfunction in Paget's disease of bone. Journal of Bone and Joint Surgery, 63B, 495.

HEATH, D.A. (1981). The role of mithramycin in the management of Paget's disease. Metabolic Bone Disease \& Related Research, 3, 343.

HERZBERG, L. \& BAYLISS, E. (1980). Spinal cord syndrome due to non-compressive Paget's disease of bone: a spinal- amelioration of bone disease. Such treatment may therefore be effective in the treatment of metastatic carcinoma of the prostate and merits further evaluation.

\section{Acknowledgements}

We are grateful for the support of the Leukaemia Research Fund, the Medical Research Council and the Special Trustees of the Former United Sheffield Hospitals. Dichloromethylene diphosphonate was kindly supplied by Istituto Gentili Spa, of Italy. R.C.P. is a Wellcome Surgical Fellow.

artery steal phenomenon reversible with calcitonin. Lancet, ii, 13.

KANIS, J.A. \& RUSSELL, R.G.G. (Eds) (1982). Diphosphonates and Paget's disease of bone. Metabolic Bone Disease \& Related Research, 3, 217.

MUNDY, A.R. (1979). Urinary hydroxyproline excretion in carcinoma of the prostate. A comparison of four different modes of assessment and its role as a marker. British Journal of Urology, 51, 570. 